\title{
Reação de germoplasma comercial de soja a Colletotrichum truncatum
}

\author{
Ivan F.D. da Costa ${ }^{1}$, Ricardo S. Balardin ${ }^{1}$, Luis A.M. Medeiros ${ }^{2}$, Giuvan Lenz ${ }^{1}$, Caroline A. Gulart ${ }^{1}$, Carla \\ R. Zemolin ${ }^{1}$ \& Tânia M.B. Silva ${ }^{1}$
}

${ }^{1}$ Departamento de Defesa Fitossanitária, Universidade Federal de Santa Maria, Centro de Ciências Rurais, 97105-900, Santa Maria, RS, Brasil; ${ }^{2}$ Centro Federal de Educação Tecnológica São Vicente do Sul, 97420-000, São Vicente do Sul, RS, Brasil

Autor para correspondência: Giuvan Lenz, e-mail: giuvanlenz@gmail.com

\section{RESUMO}

No período de março de 2004 a janeiro de 2005 foi avaliada a resistência de 48 cultivares comerciais de soja a Colletotrichum truncatum. Plântulas de soja foram inoculadas no estádio $\mathrm{V}_{1} / \mathrm{V}_{2} \mathrm{e}$, após as inoculações, foram mantidas em casa de vegetação, sob condições de umidade relativa do ar acima de $95 \%$, durante 10 dias. Após esse período, as plântulas foram avaliadas para determinar a reação das cultivares. A severidade dos sintomas foi obtida pela avaliação das lesões nas folhas, determinando-se os graus de resistência das plântulas através de uma escala de notas de severidade, com valores de 1 a 9. Somente as cultivares Tabarana, Cometa e EMGOPA 316 foram suscetíveis a Colletotrichum truncatum.

Palavras-chave: Glycine max, antracnose, resistência genética.

\begin{abstract}
Reaction of commercial germplasm of soybean to Colletotrichum truncatum

During the period between March, 2004 and January 2005, the reaction of 48 commercial cultivars of soybean to Colletotrichum truncatum was evaluated in the greenhouse facilities of the Universidade Federal de Santa Maria. The seedlings were inoculated in the stadium $\mathrm{V}_{1} / \mathrm{V}_{2}$. After inoculation, plants were kept in the greenhouse under a relative humidity of $95 \%$ for 10 days, before evaluation. After that period, the seedlings were evaluated to determine the reaction of cultivars. Seedlings were evaluated for disease reaction according to a scale with values from 1 to 9. Only the cultivars Tabarana, Cometa and Emgopa 316 showed to be susceptible to Colletotrichum truncatum.
\end{abstract}

Keywords: Glycine max, anthracnose, genetic resistance.

Embora a antracnose da soja, causada por Colletotrichum truncatum (Schwein.) Andrus \& W.D. Moore, seja considerada como uma das principais doenças da cultura na região do cerrado brasileiro, principalmente sob condições de alta umidade, quando pode causar perdas totais de produção (Balardin, 2002), poucos trabalhos têm sido realizados com o objetivo de se determinar a reação de cultivares comerciais de soja ao patógeno. Mesmo a recomendação oficial de controle da doença não trata do uso de cultivares resistentes, enfatizando apenas os métodos culturais de controle como a rotação de culturas, manejo do solo e adubação potássica balanceada, maiores espaçamentos e menor densidade de plantas, que visam diminuir a pressão de inóculo nas áreas de ocorrência da doença (Recomendações, 2007; Balardin, 2002). Os danos causados

Parte da Tese de Doutorado do primeiro autor. Universidade Federal de Santa Maria. Santa Maria RS. 2005 pelo fungo, em anos de intensa precipitação pluviométrica e temperaturas baixas durante o estabelecimento da cultura, podem resultar da morte de plântulas, em pré e pós emergência, do desfolhamento prematuro, da senescência precoce e do subdesenvolvimento das vagens e sementes (Manandhar \& Hartman, 1999).

A doença caracteriza-se por apresentar, no estádio de plântula, a chamada infecção latente, ou seja, em que o patógeno infecta a planta mas os sintomas da doença, só serão observados em estádios mais avançados do seu desenvolvimento. Klingelfuss \& Yorinori (2001), estudando a infecção latente nas folhas e nas hastes de soja, observaram que Colletotrichum truncatum e Cercospora kikuchii estavam presentes nos folíolos de soja, mesmo na ausência de sintomas no campo.

Segundo Chandrasekaran et al. (2000), cultivares resistentes e suscetíveis de soja a C. truncatum diferenciamse quanto à concentração de fenóis e de enzimas oxidantes. A soja pode apresentar diferenças de suscetibilidade à 
infeccão por C. truncatum em vários de seus órgãos, como nas folhas (Khare \& Chacko, 1983), em sementes (Hepperly et al., 1983; Khare \& Chacko, 1983), nas vagens e nos ramos (Backman et al., 1982). Testes de determinação de resistência de variedades de soja a $C$. truncatum, sob condições epifitóticas, na região de Nagpur, Índia, demonstraram que as variedades "JS-22" e "PKV1 " foram altamente resistentes ao patógeno, enquanto que a variedade "MACS-13" se mostrou apenas resistente (Ghawde et al., 1996). Com a inoculação de diferentes raças de C. lindemuthianum em diferentes cultivares de feijoeiro, foi observado o desenvolvimento de sintomas mais severos nos estádios iniciais de desenvolvimento da planta, demonstrando que a idade da planta influencia na sua suscetibilidade ao patógeno (Medeiros, 2004. Medeiros et al., 2008).

Singh (1993) avaliou a reação de 48 cultivares de soja à infecção por $C$. truncatum, em campo, com inoculação no início da formação da vagem, utilizando uma suspensão de esporos contendo $1 \times 10^{4}$ conídios $/ \mathrm{mL}$. Nenhuma das cultivares testadas se apresentou imune, entretanto, o grau de resistência variou bastante entre as cultivares, sendo "HM1" e "Birsa Soybean 2" as mais resistentes.

Espécies do gênero Colletotrichum sp., como por exemplo, C. lindemuthianum, agente causal da antracnose do feijão, possuem uma grande diversidade genética, apresentando diferentes raças fisiológicas (Barrus, 1911; Medeiros et al., 2008), capazes de atacar seus hospedeiros com maior ou menor intensidade. Como conseqüência dessa variabilidade inerente às espécies do gênero Colletotrichum sp., supõe-se que provavelmente existam reações diferencias de resistência ou suscetibilidade das cultivares frente ao patógeno. $\mathrm{O}$ objetivo deste trabalho foi avaliar a resistência de cultivares comerciais de soja a Colletotrichum truncatum.

Foram avaliadas 48 cultivares comerciais de soja, recomendadas para as diferentes regiões de plantio de soja do país. O trabalho foi conduzido em casa de vegetação, no município de Itaara RS, e na Clínica Fitossanitária do Departamento de Defesa Fitossanitária, CCR, UFSM, Santa Maria RS, no período de março de 2004 a janeiro de 2005. $\mathrm{O}$ isolado de C. truncatum utilizado foi obtido junto ao Setor de Fitopatologia da Faculdade de Agronomia Eliseu Maciel da Universidade Federal de Pelotas, RS.

O fungo foi cultivado em meio BDA ( $20 \%$ batata, $2 \%$ dextrose, $1,5 \%$ Agar), quando as colônias mostraramse com crescimento micelial com diâmetro próximo de 2 $\mathrm{cm}$, foi repicado para meio de cultura Mathur (Mathur et al., 1950). O período de incubação do isolado foi de 14 dias, em condições de fotoperíodo 12/12 h (luz/escuro) e temperatura de $21^{\circ} \mathrm{C}$. Culturas puras foram obtidas a partir de culturas monospóricas. A multiplicação do patógeno foi realizada em meio Mathur modificado, onde, sobre o meio ainda líquido, foram acrescentados pedaços de $2 \mathrm{~cm}$ de folhas de taboa (Tipha sp.), previamente autoclavadas, sobre as quais foi inoculado o isolado. Após sete dias em crescimento nas mesmas condições descritas anteriormente, pedaços desse meio de cultura, com abundante crescimento de acérvulos, foram transferidos para frascos com tampa de rosca, com capacidade para $100 \mathrm{~mL}$, contendo folhas de taboa autoclavadas, onde foi produzido o inóculo em quantidade suficiente para a inoculação.

Após 15 dias de crescimento nas mesmas condições descritas anteriormente, quando as folhas de taboa se apresentavam totalmente colonizadas pelo fungo, foi acrescentada nos frascos de vidro uma mistura de água mais espalhante Tween $80(0,01 \%)$, na concentração de $0,5 \%$ $(0,5 \mathrm{~mL}$ de Tween $80+$ água destilada e esterilizada Q.S.P. $100 \mathrm{~mL}$ ), para facilitar a liberação dos esporos. A mistura foi agitada vigorosamente para retirada dos esporos e, em seguida, foi filtrada para retirada de impurezas. Na suspensão de esporos resultante foi realizado um ajuste, com auxílio de Câmara de Neubauer, para alcançar a concentração final de $1,2 \times 10^{6}$ esporos $/ \mathrm{mL}$.

As 48 cultivares foram semeadas em delineamento experimental inteiramente ao acaso, com quarenta e oito tratamentos e dez repetições. As unidades experimentais constaram de bandejas plásticas, com aproximadamente 4 L de substrato organo-mineral, onde as plântulas de soja foram cultivadas. Em cada bandeja, foram semeadas 10 sementes de quatro cultivares, sendo considerada, uma cultivar por tratamento e cada planta correspondendo a uma repetição. A semeadura constou de 10 sementes de cada cultivar, que foram pré-germinadas em caixas Blottertest, posteriormente transplantadas para as bandejas. Essas bandejas foram colocadas em estufa plástica, com ambiente parcialmente controlado, onde as plântulas de soja foram inoculadas no estádio $V_{1} / V_{2}$ (folha primária completamente expandida), com a suspensão de esporos, que foi pulverizada sobre as plantas com um micropulverizador automotivo, regulado para uma pressão de $15 \mathrm{lb} / \mathrm{pol}^{2}$. A suspensão de esporos foi colocada sobre toda a parte aérea das plântulas, em ambas as faces das folhas, sendo evitado o escorrimento da suspensão de inóculo. Após as inoculações, as plântulas foram mantidas em casa de vegetação, sob condições de umidade relativa do ar acima de $95 \%$, durante 10 dias. O fotoperíodo aproximado foi de 10/14 h (luz/escuro) com a temperatura variando entre 18 e $22^{\circ} \mathrm{C}$. Após o período de 10 dias, as plântulas foram avaliadas para determinar a reação das cultivares.

A avaliação da reação das plântulas foi feita com base em uma escala de notas de severidade de doença, com as seguintes atribuições: 0 (sem sintomas); 0,1-1,0 (lesões $<1 \mathrm{~mm}$ no pecíolo e/ou nervuras); 1,1-2,0 (poucas lesões $<3 \mathrm{~mm}$ limitadas ao pecíolo); 2,1-3,0 (poucas lesões $<3$ $\mathrm{mm}$ limitadas às nervuras); 3,1-4,0 (numerosas lesões $<3$ $\mathrm{mm}$ limitadas às nervuras e/ou pecíolos); 4,1-5,0 (lesões no pecíolo e/ou nervuras $>3 \mathrm{~mm} \mathrm{e}<5 \mathrm{~mm}$ ); 5,1-6,0 (lesões no pecíolo e/ou nervuras $>5 \mathrm{~mm}$ e $<10 \mathrm{~mm}$ ); 6,1-7,0 (lesões $>10 \mathrm{~mm}$ no pecíolo e/ou nervuras); 7,1-8,0 (lesões $>10$ $\mathrm{mm}$ no pecíolo e/ou nervuras, com esporulação) e 8,1-9,0 (folhas mortas). As cultivares foram separadas em três 
grupos de reação distinta: I) reação resistente: às quais foram atribuídas notas de 0,1 a 3,0; II) reação intermediária: às quais foram atribuídas notas de 3,1 a 6,0 e III) reação suscetível: com notas de 6,1 a 9,0. Foi realizada a análise de variância dos dados de severidade e as médias comparadas pelo Teste de Scott-Knott, a 5\% de probabilidade de erro.

Sabe-se que o desenvolvimento de cultivares resistentes é uma estratégia de grande importância no manejo integrado de doenças, visto que apresenta reduzido impacto ambiental, baixo custo e limitada necessidade de adoção de insumos para o controle de doenças. A determinação da resistência juvenil é um dado importante para a seleção de genótipos em programas de melhoramento genético, já que embora $C$. truncatum mostre seus sintomas sobre a soja a partir do estádio de pré-floração e início da floração (Manandhar \& Hartman, 1999) é reconhecido seu dano em plântulas de soja durante os estádios $\mathrm{V}_{1} \mathrm{e} \mathrm{V}_{2}$, causando tombamento. A análise de variância mostrou a existência de diferenças significativas $(\mathrm{p} \leq 0,05)$ entre as cultivares, devido à ação do isolado de $C$. truncatum (Pel1) inoculado sobre as cultivares de soja testadas, quando agrupadas em função do ciclo vegetativo (Tabela 1). As cultivares apresentaram três grupos de reação distinta: I) reação resistente: com 18 cultivares; II) reação intermediária: com 28 cultivares e III) reação suscetível: com 3 cultivares. Nenhuma cultivar apresentou reação de imunidade (nota 0 ). As condições de incubação das cultivares após a inoculação, proporcionaram um ambiente favorável ao desenvolvimento do fungo, que em condições de campo, causa danos às plantas sob temperaturas entre 23 e $30^{\circ} \mathrm{C}$, umidade relativa e períodos de molhamento foliar altos (Singh et al., 2001; Chongo \& Bernier, 2000). Ghawde et al. (1996), em trabalho de identificação de genótipos de soja resistentes a C. truncatum, demonstraram a ocorrência de cultivares com altos graus de resistência onde, sob condições ideais ao desenvolvimento do patógeno, em casa de vegetação, um grupo significativo de cultivares se mostrou resistente (37\% das cultivares) ou com reação intermediária ( $57 \%$ das cultivares).

As cultivares do grupo de ciclo precoce Vencedora, Embrapa 48, BRS 133, BRS Macota, CD 201, Iguaçú,

TABELA 1 - Reação de cultivares de soja do grupo de ciclo precoce, médio e tardio ao isolado de Colletotrichum truncatum (Pel1), inoculado no estádio $\mathrm{V}_{1} / \mathrm{V}_{2}$. Santa Maria RS, 2005

\begin{tabular}{|c|c|c|c|c|c|c|c|c|c|c|c|}
\hline \multicolumn{4}{|c|}{ Grupo de ciclo precoce } & \multicolumn{4}{|c|}{ Grupo de ciclo médio } & \multicolumn{4}{|c|}{ Grupo de ciclo tardio } \\
\hline Cult. $^{1}$ & Cic. & Sev. & Rea. & Cult. & Cic. & Sev. & Rea. & Cult. & Cic. & Sev. & Rea. \\
\hline Tabarana & $\mathrm{P}^{2}$ & $7,70 \mathrm{a}^{3}$ & $\mathrm{~S}^{4}$ & Santa Cruz & M & $4,30 \mathrm{a}$ & I & CD 205 & ST & $5,90 \mathrm{a}$ & I \\
\hline Cometa & $\mathrm{P}$ & $6,90 \mathrm{~b}$ & $\mathrm{~S}$ & CEP 33 & M & $4,30 \mathrm{a}$ & I & FEPAGRO 23 & $\mathrm{~T}$ & $4,90 \mathrm{~b}$ & I \\
\hline Emgopa 316 & $\mathrm{P}$ & $6,20 \mathrm{~b}$ & $\mathrm{~S}$ & MSOY 7501 & M & $4,10 \mathrm{a}$ & I & JATAÍ & $\mathrm{T}$ & $4,10 \mathrm{c}$ & I \\
\hline CD 207 & $\mathrm{P}$ & $5,80 \mathrm{c}$ & I & MSOY 7901 & M & $3,90 \mathrm{a}$ & I & IAC 19 & $\mathrm{~T}$ & $3,60 \mathrm{~d}$ & I \\
\hline MSOY 5942 & $\mathrm{P}$ & $5,50 \mathrm{c}$ & I & Emgopa 315 & M & $3,60 \mathrm{~b}$ & I & FEPAGRO RS & $\mathrm{T}$ & $3,50 \mathrm{~d}$ & I \\
\hline CD 208 & $\mathrm{P}$ & $5,40 \mathrm{c}$ & I & BRS 154 & M & $3,50 \mathrm{~b}$ & I & EMGOPA 313 & $\mathrm{~T}$ & $3,20 \mathrm{~d}$ & I \\
\hline CD 202 & $\mathrm{P}$ & $5,40 \mathrm{c}$ & I & BRS Luziânia & M & $3,40 \mathrm{~b}$ & I & BRS TORENA & ST & $3,20 \mathrm{~d}$ & I \\
\hline CD 215 & $\mathrm{P}$ & $4,60 \mathrm{~d}$ & I & CEP 39 & M & $3,20 \mathrm{~b}$ & I & IAC 82 & $\mathrm{~T}$ & $2,90 \mathrm{~d}$ & $\mathrm{R}$ \\
\hline Icasc 1 & SPP & $4,60 \mathrm{~d}$ & I & CEP 38 & M & $3,10 \mathrm{~b}$ & I & Sambaíba & $\mathrm{T}$ & $2,80 \mathrm{~d}$ & $\mathrm{R}$ \\
\hline Liderança & SP & $4,10 \mathrm{e}$ & I & MSOY 8400 & M & $2,80 \mathrm{~b}$ & $\mathrm{R}$ & FT Abyara & $\mathrm{T}$ & $2,20 \mathrm{e}$ & $\mathrm{R}$ \\
\hline IAS-5 & $\mathrm{P}$ & $4,10 \mathrm{e}$ & I & Conquista & M & $2,00 \mathrm{c}$ & $\mathrm{R}$ & Garantia & ST & $2,20 \mathrm{e}$ & $\mathrm{R}$ \\
\hline MSOY 6101 & $\mathrm{P}$ & $4,00 \mathrm{e}$ & I & BRS 153 & M & $2,00 \mathrm{c}$ & $\mathrm{R}$ & & & & \\
\hline BRS 138 & $\mathrm{P}$ & $3,80 \mathrm{e}$ & I & & & & & & & & \\
\hline CD 217 & SP & $3,70 \mathrm{e}$ & I & & & & & & & & \\
\hline V-MAX & $\mathrm{P}$ & $3,70 \mathrm{e}$ & I & & & & & & & & \\
\hline Vencedora & $\mathrm{SP}$ & $3,00 \mathrm{f}$ & $\mathrm{R}$ & & & & & & & & \\
\hline Embrapa 48 & $\mathrm{P}$ & $3,00 \mathrm{f}$ & $\mathrm{R}$ & & & & & & & & \\
\hline BRS 133 & $\mathrm{SP}$ & $2,90 \mathrm{f}$ & $\mathrm{R}$ & & & & & & & & \\
\hline BRS Macota & $\mathrm{P}$ & $2,80 \mathrm{f}$ & $\mathrm{R}$ & & & & & & & & \\
\hline CD 201 & $\mathrm{P}$ & $2,80 \mathrm{f}$ & $\mathrm{R}$ & & & & & & & & \\
\hline Iguaçu & $\mathrm{P}$ & $2,70 \mathrm{f}$ & $\mathrm{R}$ & & & & & & & & \\
\hline BRS 184 & $\mathrm{P}$ & $2,50 \mathrm{f}$ & $\mathrm{R}$ & & & & & & & & \\
\hline CD 204 & $\mathrm{P}$ & $2,50 \mathrm{f}$ & $\mathrm{R}$ & & & & & & & & \\
\hline MSOY 8001 & SP & $2,40 \mathrm{f}$ & $\mathrm{R}$ & & & & & & & & \\
\hline IAC 18 & $\mathrm{P}$ & $2,30 \mathrm{f}$ & $\mathrm{R}$ & & & & & & & & \\
\hline C.V. & & 20,58 & & C.V. & & 23,43 & & C.V. & & 22,70 & \\
\hline
\end{tabular}

${ }^{1}$ Cult.: Cultivar utilizada; Cic.: Ciclo; Sev.: Severidade da doença; Rea.: Reação genética; ${ }^{2}$ P: precoce; SP: semi-precoce; SPP: super-precoce; M: médio; T: tardio; ST: semi-tardio; C.V.: coeficiente de variação; ${ }^{3}$ Médias seguidas da mesma letra não diferem pelo Teste de Scott-Knott, ao nível de $5 \%$ de probabilidade de erro; ${ }^{4} \mathrm{~S}$ : suscetível; R: resistente; I: intermediário. 
BRS 184, CD 204, MSOY 8001 e IAC 18 apresentaram reação de resistência à inoculação pelo isolado de $C$. truncatum testado, sendo materiais promissores para utilização em programas de controle integrado de doenças. As cultivares Tabarana, Cometa e EMGOPA 316 foram as únicas a apresentar reação de suscetibilidade entre todas as cultivares testadas, e desta forma a sua utilização deve ser feita associada a um programa de controle onde seja dado ênfase ao tratamento de sementes, principalmente naqueles locais onde existe histórico de ocorrência de infecção por C. truncatum. Entre as cultivares do grupo de ciclo médio, não ocorreram reações de suscetibilidade, e embora apenas as cultivares MSoy 8400, Conquista e BRS 153 tenham apresentado reação resistente, todas as demais apresentaram notas inferiores a estas, permanecendo no grupo de reação intermediária. No grupo de ciclo tardio, as cultivares IAC 82, Sambaíba, FT Abyara e Garantia foram as que apresentaram reação resistente, sendo as demais definidas como intermediárias, mas com a cultivar CD 205 muito próxima do limiar do grupo de suscetibilidade. Com base nos critérios de definição das classes de reação podese concluir que apenas as cultivares Tabarana, Cometa e EMGOPA 316 foram suscetíveis a Colletotrichum truncatum (isolado Pel1), sendo as demais consideradas resistentes ou intermediárias.

\section{REFERÊNCIAS BIBLIOGRÁFICAS}

Backman PA, Williams JC, Crawford MA (1982) Yield losses in soybeans from anthracnose caused by Colletotrichum truncatum. Plant Disease 66:1032-1034.

Balardin RS (2002) Doenças da Soja. Santa Maria RS. Edição do Autor.

Barrus MF (1911) Variation of cultivars of beans in their susceptibility to anthracnose. Phytopathology 1:190-195.

Chandrasekaran A, Nasasimhan V, Pajappan K (2000) Integrated management of anthracnose and pod blight of soybean. Annals of Plant Protection Science 8:163-165.
Chongo G, Bernier CC (2000) Disease incidence, lesion size, and sporulation in Colletotrichum truncatum as influenced by lentil genotype and temperature. Canadian Journal of Plant Pathology 22:236-240.

Ghawde RS, Gaikwad SJ, Borkar SL (1996) Evaluation of fungicides and screening of varieties against pod blight nof soybean caused by Colletotrichum truncatum (Schw.). Andus and Moore. Journal of Soil and Crops 6:97-99.

Hepperly PR, Mignucci JS, Sinclair JB, Mendoza JB (1983) Soybean anthracnose and its seed assay in Puerto Rico. Seed Science and Technology 11:371-380.

Khare MN, Chacko S (1983) Factors affecting seed infection and transmission of Colletotrichum dematium f.sp. truncata in soybean. Seed Science and Technology 11:853-858.

Klingelfuss LH, Yorinori JT (2001) Infecção latente de Colletotrichum truncatum e Cercospora kikuchii em soja. Fitopatologia Brasileira 26:158-164.

Manandhar JB, Hartman GL (1999) Anthracnose. In: Hartman GL, Sinclair JB, Rupe JC (Eds.) Compendium of soybean diseases. 4. ed. Saint Paul MN. APS Press. pp. 13-14.

Mathur RS, Barnett HL, Lilly VG (1950) Sporulation of Colletotrichum lindemuthianum in culture. Phytopathology 40:104-114

Medeiros LAM (2004) Resistência genética do feijão (Phaseolus vulgaris L.) ao Colletotrichum lindemuthianum. Tese de Doutorado. Santa Maria RS. Universidade Federal de Santa Maria.

Medeiros LAM, Balardin RS, Costa IFD, Gulart CA, Lenz G (2008) Reação de germoplasma crioulo de feijoeiro (Phaseolus vulgaris L.) a Colletotrichum lindemuthianum. Tropical Plant Pathology 33:273-280.

Recomendações (2007) 35 $5^{\text {a }}$ Reunião da Pesquisa de Soja da Região Sul. Santa Maria. Indicações Técnicas para a Cultura da Soja no Rio Grande do Sul e em Santa Catarina 2007/2008. Santa Maria RS. Ed. Orium.

Singh DP (1993) Relative susceptibility of soybean cultivars to pod blight by Colletotrichum truncatum (Schw.). Agricultural Science Digest 13:90-92.

Singh R, Singh SB, Singh PN (2001) Effect of envirommental conditions on development of anthracnose of soybean. Annals of Plant Protection Sciences 9:146-147.

Recebido 17 Julho 2008 - Aceito 10 Fevereiro 2009 - TPP 8081 Editor Associado: Carlos R. Casela 